\title{
Altered brain activity in patients with strabismus and amblyopia detected by analysis of regional homogeneity: A resting-state functional magnetic resonance imaging study
}

\author{
YI SHAO $^{1 *}$, QING-HAI LI ${ }^{*}$, BIAO LI ${ }^{1}$, QI LIN ${ }^{1}$, TING SU $^{2}$, WEN-QING SHI ${ }^{1}$, PEI-WEN ZHU ${ }^{1}$, \\ QING YUAN ${ }^{1}$, YONG-QIANG SHU ${ }^{3}$, YING HE ${ }^{1}$, WEN-FENG LIU ${ }^{1}$ and LEI YE ${ }^{1}$ \\ ${ }^{1}$ Department of Ophthalmology, The First Affiliated Hospital of Nanchang University, \\ Jiangxi Province Ocular Disease Clinical Research Center, Nanchang, Jiangxi 330006; \\ ${ }^{2}$ Eye Institute of Xiamen University, Fujian Provincial Key Laboratory of Ophthalmology and Visual Science, \\ Xiamen, Fujian 361000; ${ }^{3}$ Department of Radiology, The First Affiliated Hospital of Nanchang University, \\ Jiangxi Province Medical Imaging Research Institute, Nanchang, Jiangxi 330006, P.R. China
}

Received August 6, 2018; Accepted March 27, 2019

DOI: $10.3892 / \mathrm{mmr} .2019 .10147$

\begin{abstract}
Previous studies have demonstrated that strabismus or amblyopia can result in marked brain function and anatomical alterations. However, differences in spontaneous brain activity in strabismus and amblyopia (SA) patients as compared with control individuals remain unclear. The present study aimed to analyze the potential brain activity changes in SA patients and their association with behavioral performance. In total, 16 patients with SA (10 women and 6 men) and 16 healthy controls (HCs; 6 men and 10 women) with matched age and sex were recruited. All subjects were examined with resting-state functional magnetic resonance imaging (rs-fMRI), and changes in the spontaneous brain activity of SA patients were evaluated by the regional homogeneity (ReHo) method. The diagnostic ability of the ReHo method was assessed using receiver operating characteristic (ROC) curve analysis. In addition, the association between the mean ReHo value in different brain regions and the behavioral performance was explored by correlation analysis. It was observed that the ReHo value was significantly increased in SA patients compared with $\mathrm{HCs}$ in the following brain regions: left lingual gyrus, right middle occipital gyrus/precuneus, bilateral anterior cingulate, left middle occipital gyrus and bilateral precentral gyrus. By contrast, the ReHo value of the
\end{abstract}

Correspondence to: Dr Lei Ye, Department of Ophthalmology, The First Affiliated Hospital of Nanchang University, Jiangxi Province Ocular Disease Clinical Research Center, 17 Yongwaizheng Street, Donghu, Nanchang, Jiangxi 330006, P.R. China

E-mail: 534616847@qq.com

*Contributed equally

Key words: strabismus with amblyopia, regional homogeneity, resting-state functional magnetic resonance imaging left inferior frontal gyrus was significantly lower than that in HCs. ROC curve analysis indicated that the ReHo method has certain credibility for the diagnosis of SA patients. In addition, no similar changes were detected in other brain regions. These results revealed abnormal spontaneous brain activity in certain parts of the brain in adult patients with SA, which suggests the involvement of the neuropathological or compensatory mechanism in these patients, and may be beneficial for clinical treatment.

\section{Introduction}

Strabismus is an ocular disease characterized by the inability of both eyes of an individual to gaze at a target simultaneously and the optic axis of both eyes is separated. It can lead to binocular vision impairment, and is often accompanied by amblyopia and loss of stereo vision. The incidence of strabismus in children aged 3-6 years in eastern China is $5.65 \%$ (1). There is no ideal classification method for strabismus in clinical practice, and its treatment mainly relies on surgical correction. Dysfunction of the extraocular muscles (EOMs) is an important cause of strabismus. In particular, aberrant EOM development, EOM dystrophy (2) and abnormal position of the EOM (3) can lead to the occurrence of strabismus, while dysfunction of the EOM pulleys has also been associated with the condition $(4,5)$. The eye movement system is underpinned by a complex neural network. The structures in the nucleus of the brain, and the EOMs and nerves that dominate them must all work perfectly and synchronously to ensure normal visual function. For instance, the development of Duane's retraction syndrome has been reported to be associated with mechanical abnormalities of the external rectus muscle (6), aberrant innervation (7) and the absence of an abduction nucleus (8). Neuronal activity in the brain area that is involved in eye movements is also crucial. The frontal eye field (FEF) participates in the control of eye movement (4) and conjugate eye movement (5). A previous study reported that the gray matter volume of the FEF is increased in strabismus patients (9). Therefore, strabismus 
pathogenesis is directly associated with the EOM and brain function and/or structural alterations.

Amblyopia is caused by abnormal visual experiences (such as monocular strabismus, anisometropia, high ametropia and form-deprivation myopia) during visual development, resulting in decreased monocular and/or binocular best-corrected visual acuity (VA), but no organic lesion is observed on eye examination. The diagnostic criteria for amblyopia include a visual reference value that is below the lower normal limit (which is 0.5 for 3-5-year-old children, and 0.7 for children aged $\geq 6$ years), or the best corrected VA of two eyes differs by $\geq 0.2$, then the eye with poorer VA exhibits amblyopia (10). In China, the prevalence of amblyopia in children is $2-3 \%$ (11). At present, early detection and early treatment of amblyopia are important, with standard treatment strategies including accurate glasses and concealment of the dominant eye. According to its etiology, amblyopia can be divided into the strabismus, anisometropic, ametropic and form-deprivation types (12). The pathogenesis of amblyopia is highly complex; the two theories currently recognized involve abnormal binocular interaction and form deprivation $(13,14)$. Simultaneously, abnormal activities in the associated brain regions have also been suggested to contribute to the development of amblyopia. For instance, Wang et al (15) reported that activation of the intraparietal sulcus, FEF and motor sensitive (V5) areas was reduced in patients with amblyopia. Thus, the exploration of brain activity in patients with strabismus and amblyopia (SA) is of practical significance.

Functional magnetic resonance imaging (fMRI) (16) is the primary examination technique used to locate and quantify functional brain areas. Its main advantages over traditional MRI techniques are high spatial resolution and the ability to reveal detailed microscopic structural changes. fMRI technology includes diffusion weighted imaging (DWI), diffusion tensor imaging (DTI), magnetic resonance spectroscopy, and blood oxygen level-dependent (BOLD) fMRI. Among these, DWI can reflect the diffusion movement and limitation of water molecules in tissues and lesions, while DTI reveals the anisotropy of diffusion movement towards water molecules, and can therefore be used to analyze white matter fiber bundles. In addition, BOLD fMRI uses the principle of blood oxygenation level dependence, that is, inconsistencies in the local hemodynamics of neurons following excitation, in order to reveal spontaneous neuronal activity by quantifying alterations in blood oxygen level signals. Thus, the study of brain cognitive activities, and the localization and quantification of brain functional activity areas is facilitated by fMRI. This technology has been widely used to study SA. In addition to these aforementioned studies, the volume of gray matter in the occipital eye field and parietal eye field has been reported to be reduced in patients with strabismus (17), while the functional connectivity between the $\mathrm{V} 1$ and $\mathrm{V} 2$ regions was decreased in SA monkeys (18). Furthermore, multiple brain areas are reportedly dysfunctional in concomitant strabismus (19).

Resting-state fMRI (rs-fMRI) analysis can be conducted using various methods, among which regional homogeneity ( $\mathrm{ReHo}$ ) is widely used. ReHo is a computational method based on functional differentiation, which was first proposed by the Chinese scholar Professor Yu-Feng Zang. This method can be used to analyze the consistency of brain activity signals and provide information about brain function $(20,21)$. ReHo functions by assuming that the hemodynamics of each voxel in a brain region with the same function is approximately identical, and that the hemodynamics of the brain region may change due to variations in function or task. Thus, the level of consistency of BOLD signals can be represented by evaluating the degree of hemodynamic consistency between voxels in the region of interest and voxels that are adjacent to it simultaneously, which can be expressed using a ReHo value that reveals the consistency of spontaneous neuronal activity. Therefore, changes in ReHo values indicate alterations in brain hemodynamics, that is, changes in the synchrony of spontaneous neuronal activity. An increase in ReHo indicates an increase in the synchrony of spontaneous neuronal activity, whereas a decreased ReHo value suggests reduced synchrony and disordered activity. This method has been successfully applied to the investigation of the etiology of various eye diseases, including concomitant strabismus (19), optic neuritis (22), type 2 diabetes with retinopathy (23), glaucoma (24), open-globe injury (25), late monocular blindness (26) and retinal detachment (27), as well as a number of neurogenic diseases, such as sleep disorders (28) and Parkinson's disease (29).

In the present study, the brain activity of patients with SA was investigated using the ReHo method, in order to confirm alterations in brain function and explore the potential pathophysiological mechanism. To the best of our knowledge, this is the first study to examine SA using this method.

\section{Subjects and methods}

Subjects. A total of 16 patients with SA who were treated in the Department of Ophthalmology of The First Affiliated Hospital of Nanchang University (Nanchang, China) were enrolled into the present study. The patients included 6 men and 10 women, among which there were 11 cases with exotropia and 5 with esotropia. The inclusion criteria were as follows: i) adults with an age of $>18$ years; ii) patients diagnosed with strabismus; and iii) >2-line difference in the best-corrected VA $(\geq 0.20 \log$ MAR units) between the amblyopic and the fellow eye, with central fixation. Patients were excluded according to the following criteria: i) patients with a history of previous ocular surgery, including intraocular and extraocular surgery; ii) patients with evidence of other eye diseases, such as cataract, glaucoma, optic neuritis, macular degeneration, infection, inflammation, and ischemic disease; iii) other diseases that may affect the experimental results, including mental illness; and iv) alcoholism or drug addiction.

In addition, 16 healthy controls (HCs), including 6 men and 10 women, were matched with the patients in terms of age and sex. All HCs met the following criteria: i) MRI examination exhibited no abnormalities in the brain parenchyma; ii) no history of eye disease, with best-corrected VA of $\leq 0 \log$ MAR units; iii) no psychiatric disease; and iv) able to undergo MRI examination (for example, no implanted steel plate).

The present study was approved by the Medical Ethics Committee of the First Affiliated Hospital of Nanchang University and conformed to all the principles required by the Declaration of Helsinki. Prior to signing informed consent, detailed information on the study was provided to participants, 
including the purpose of the research and possible risks to subjects.

MRI parameters. All subjects were scanned with a 3-Tesla magnetic resonance scanner (Trio; Siemens AG, Munich, Germany). All participants were requested to stay awake, keep their eyes closed and relax their body until the end of the scan. Conventional T1 weighted image (T1WI) scans were collected, as well as T2WI structural magnetic resonance data for the exclusion of brain structural lesions, 3D high-resolution T1WI volume image data and rs-fMRI data. Among them, 176 3D high-resolution T1WI volume images were acquired by the T1-weighted 3D spoiled gradient sequence (30). The specific scanning parameters used were as follows: repetition time (TR), 1,900 msec; echo time (TE), 2.26 msec; thickness, $1.0 \mathrm{~mm}$; gap, $0.5 \mathrm{~mm}$; acquisition matrix, 256x256; flip angle, $9^{\circ}$; field-of-view (FOV), 250×250 mm. Furthermore, a total of 240 functional images were acquired using the gradient-recalled echo-planar imaging sequence (31), according to the following specific scanning parameters: TR, 2,000 msec; TE, $30 \mathrm{msec}$; thickness, $4.0 \mathrm{~mm}$; gap, $1.2 \mathrm{~mm}$; acquisition matrix, 64x64; flip angle, $90^{\circ}$; and FOV, $220 \times 220 \mathrm{~mm}$. The scanning times for the two sequences were 5 and $10 \mathrm{~min}$, respectively.

fMRI data processing. Initially, the MRICRO software package (http://www.MRIcro.com) was used to examine and screen the acquired brain data. Next, the SPM8 software package (http://www.fil.ion.ucl.ac.uk/spm) on the Matlab R2012b platform (MathWorks, Natick, MA, USA) was applied to preprocess the two sets of data in this experiment, using the data processing software package DPARSF (http://rfmri. org/DPARSF). The main steps of the analysis were as follows: first, the data format was converted. In order to remove the interference of the unstable magnetic field, the data of the first 10 time points were then eliminated. In order to remove the impact caused by different data acquisition time, time correction was subsequently performed on the collected data. Next, head movement correction was performed; head movement was considered to be too large and the data was eliminated for one case that had a maximum head movement in three directions of $>2 \mathrm{~mm}$ and a maximum rotation angle of $>2^{\circ}$. Subsequently, spatial standardization of the functional image was conducted, which was necessary since each subject has a certain difference in brain structure and volume. For this standardization, the functional image was registered to the standard space of the Montreal Neuroscience Institute, and all voxels were resampled to a size of $3 \times 3 \times 3 \mathrm{~mm}$ to obtain a more accurate brain area. The next step involved removal of linear drift in order to eliminate the linear chemotactic effect of the subject in the process of adapting to the scanning environment. Finally, filtering was performed by collecting data in the frequency range of $0.01-0.08 \mathrm{~Hz}$ to remove the influence of high-frequency physiological noise, such as breathing and heartbeat.

ReHo calculation and image processing. REST software (http://sourceforge.net/projects/testing-fmri) was used to calculate ReHo values for fMRI data without smooth preprocessing. ReHo images were derived from the Kendall consistency coefficient, which was obtained using the DPABI toolkit software (http://rfmri.org/DPABI) to calculate the time series consistency of each voxel in the brain and 26 voxels adjacent to it. Next, the image was normalized, and the Fisher $r$ to $\mathrm{Z}$ transformation was performed. Finally, a 6x6x6 mm full-width was applied at half-maximum Gaussian smooth kernel to perform Gaussian smoothing of the ReHo image in order to improve the signal-to-noise ratio.

Statistical analysis. Statistical evaluation of differences in variables, such as demographics and visual measurements, between patients and normal controls were conducted in SPSS version 20.0 software (IBM Corp., Armonk, NY, USA) using two-sample t-tests. Differences in ReHo values between SA and HC subjects were evaluated using two-sample t-tests in REST software (State Key Laboratory of Cognitive Neuroscience and Learning, Beijing Normal University, Beijing, China). At the voxel level, the statistical threshold was set to $\mathrm{P}<0.05$, and for multiple comparisons using Gaussian random field theory voxels, thresholds of $\mathrm{P}<0.01$ and cluster size of $>40$ voxels (AlphaSim-corrected) were employed. Using differences in ReHo values in the same brain regions, receiver operating characteristic (ROC) curves were generated to analyze and identify SA patients and HCs. Pearson correlation analysis was also used to evaluate the correlation between ReHo values and clinical features of patients with altered brain regions, including the association between disease duration and ReHo values. Correlations and differences between SA and HC subjects were considered to be statistically significant at $\mathrm{P}<0.05$.

\section{Results}

Demographics and visual measurements. No significant differences in age $(\mathrm{P}=0.615)$ or best-corrected VA of the fellow eye $(\mathrm{P}=0.185)$ were detected between the two groups. By contrast, the differences observed between the two groups in the best-corrected VA of the amblyopic eye were statistically significant $(\mathrm{P}<0.001$; Table I).

ReHo differences. In the SA group, ReHo values were significantly increased relative to the HCs in the following brain regions: left lingual gyrus (LLG), right middle occipital gyrus and right precuneus (RMOG/RP), bilateral anterior cingulate (BAC), left middle occipital gyrus (LMOG) and bilateral precentral gyrus (BPG) [Fig. 1 (red shading) and Table II)]. By contrast, the ReHo values of the left inferior frontal gyrus (LIFG) were markedly reduced in SA patients as compared with those in HCs [Fig. 1 (blue shading) and Table II]. The mean ReHo values in the two groups are presented in Fig. 2. The ReHo values in the altered brain areas of SA patients were not significantly associated with any of the clinical features evaluated in the present study (Table III).

ROC curve analysis. By computational analysis, it was observed that the ReHo values of altered brain regions may be useful diagnostic indicators for SA (Table II). To verify these findings, ROC curves were generated to analyze the ReHo values in brain regions exhibiting apparent differences in SA patients. Area under the curve (AUC) values of 0.7-0.9 indicates that the disease can be diagnosed more accurately. The individual AUCs of ReHo values in the different regions were as follows: LLG, AUC $=0.934(\mathrm{P}<0.001) ; \mathrm{RMOG} / \mathrm{RP}, \mathrm{AUC}=0.965(\mathrm{P}<0.001)$; 
Table I. Demographics and clinical measurements of SA and HC groups.

\begin{tabular}{|c|c|c|c|c|}
\hline Parameter & SA & $\mathrm{HC}$ & t-value & $\mathrm{P}$-value \\
\hline Male/female & $6 / 10$ & $6 / 10$ & - & $>0.99$ \\
\hline Age (years) & $24.50 \pm 5.91$ & $24.94 \pm 5.23$ & -0.222 & 0.615 \\
\hline Handedness & $16 \mathrm{R}$ & $16 \mathrm{R}$ & - & $>0.99$ \\
\hline Disease duration (years) & $18.19 \pm 9.85$ & - & - & - \\
\hline Esotropia/exotropia & $5 / 11$ & - & - & - \\
\hline Spherical equivalent refractive error (diopters) & $\begin{array}{r}1.22 \pm 0.56 \\
(-2.75-1.75)\end{array}$ & $\begin{array}{c}1.25 \pm 0.67 \\
(-2.75-2.00)\end{array}$ & -0.365 & 0.741 \\
\hline Angle of strabismus (PD) & $26.25 \pm 12.71$ & - & - & - \\
\hline \multicolumn{5}{|l|}{ Best-corrected VA } \\
\hline Amblyopic eye & $0.77 \pm 0.53$ & $-0.05 \pm 0.08$ & 6.149 & $<0.001$ \\
\hline Fellow eye & $-0.03 \pm 0.09$ & $-0.01 \pm 0.07$ & -0.651 & 0.185 \\
\hline
\end{tabular}

Independent t-tests comparing the two groups $(\mathrm{P}<0.05$ represented statistically significant differences). Data shown as mean standard deviation or n. SA, strabismus and amblyopia; HC, healthy control; N/A, not applicable; PD, prism diopter; VA, visual acuity; R, right.

Table II. Brain areas with significantly different ReHo values between groups.

\section{A, SA $>\mathrm{HC}$}

\begin{tabular}{|c|c|c|c|c|c|c|c|c|}
\hline \multirow[b]{2}{*}{ Condition } & \multirow[b]{2}{*}{ Left/right } & \multirow[b]{2}{*}{ Brain regions } & \multirow[b]{2}{*}{$\mathrm{BA}$} & \multicolumn{3}{|c|}{ MNI coordinates } & \multirow[b]{2}{*}{ Peak voxels } & \multirow[b]{2}{*}{ t-value } \\
\hline & & & & $\mathrm{X}$ & Y & $\mathrm{Z}$ & & \\
\hline 1 & Left & Lingual gyrus & 19 & -36 & -69 & -9 & 86 & 3.6987 \\
\hline 2 & Right & Middle occipital gyrus/precuneus & 19 & 33 & -84 & 12 & 256 & 4.7141 \\
\hline 3 & Bilateral & Anterior cingulate & - & 6 & 30 & -6 & 74 & 3.8637 \\
\hline 4 & Left & Middle occipital gyrus & 19 & -27 & -87 & 9 & 116 & 4.5112 \\
\hline 5 & Bilateral & Precentral gyrus & 6 & 0 & -6 & 78 & 339 & 5.5492 \\
\hline
\end{tabular}

$\mathrm{B}, \mathrm{SA}<\mathrm{HC}$

\begin{tabular}{|c|c|c|c|c|c|c|c|c|}
\hline \multirow[b]{2}{*}{ Condition } & \multirow[b]{2}{*}{ Left/right } & \multirow[b]{2}{*}{ Brain regions } & \multirow[b]{2}{*}{ BA } & \multicolumn{3}{|c|}{ MNI coordinates } & \multirow[b]{2}{*}{ Peak voxels } & \multirow[b]{2}{*}{ t-value } \\
\hline & & & & $\mathrm{X}$ & $\mathrm{Y}$ & $\mathrm{Z}$ & & \\
\hline 1 & Left & Inferior frontal gyrus & 9 & -51 & 18 & 24 & 69 & -4.0693 \\
\hline
\end{tabular}

$\mathrm{BAC}, \mathrm{AUC}=0.902(\mathrm{P}<0.001) ; \mathrm{LMOG}, \mathrm{AUC}=0.938(\mathrm{P}<0.001)$; $\mathrm{BPG}, \mathrm{AUC}=0.922(\mathrm{P}<0.001) ;$ and LIFG, AUC $=0.938(\mathrm{P}<0.001$; Fig. 3). Taken together, these findings suggest that the ReHo values of altered brain regions may serve as diagnostic indicators for SA.

\section{Discussion}

rs-fMRI is easier to implement in patients than task-based fMRI, since patients are not required to perform specific tasks during fMRI scans, thus reducing the potential influence of confounding factors on the process (16). rs-fMRI can also provide more functional information, helping to better understand the functional mechanisms underlying specific diseases (16). The ReHo method has been successfully applied in several ophthalmological and neurogenic diseases, and has huge potential for further development (Table IV). To the best of our knowledge, the present study is the first to evaluate resting-state brain activity of patients with SA using the ReHo technique. 
Table III. Pearson correlations analysis.

\begin{tabular}{|c|c|c|c|c|}
\hline Brain regions & $\begin{array}{l}\text { ReHo value } \\
(\text { mean } \pm \mathrm{SD})\end{array}$ & $\begin{array}{l}\text { Duration (years) } \\
(\text { mean } \pm \mathrm{SD})\end{array}$ & r-value & P-value \\
\hline Left lingual gyrus & $0.6316 \pm 0.4937$ & $18.05 \pm 9.55$ & -0.343 & 0.196 \\
\hline Right middle occipital gyrus/precuneus & $0.8429 \pm 0.3633$ & & -0.254 & 0.342 \\
\hline Bilateral anterior cingulate & $0.2272 \pm 0.4586$ & & 0.063 & 0.817 \\
\hline Left middle occipital gyrus & $0.8784 \pm 0.4560$ & & -0.342 & 0.195 \\
\hline Bilateral precentral gyrus & $0.3208 \pm 0.2700$ & & -0.360 & 0.171 \\
\hline Left inferior frontal gyrus & $-0.0320 \pm 0.3511$ & & -0.497 & 0.050 \\
\hline
\end{tabular}

ReHo, regional homogeneity; SD, standard deviation.

Table IV. ReHo method applied in ophthalmological and neurogenic diseases in the literature.

\begin{tabular}{lll}
\hline Author & Year & Disease \\
\hline Ophthalmological diseases & & \\
Song et al & 2014 & Glaucoma \\
Cui et al & 2014 & Diabetic retinopathy \\
Shao et al & 2015 & Optic neuritis \\
Huang et al & 2016 & Concomitant strabismus \\
Huang et al & 2016 & Open-globe injury \\
Huang et al & 2017 & Late monocular blindness \\
Huang et al & 2017 & Retinal detachment \\
Neurogenic diseases & & \\
Dai et al & 2012 & Sleep disorders \\
Li et al & 2016 & Parkinson's disease \\
\hline
\end{tabular}

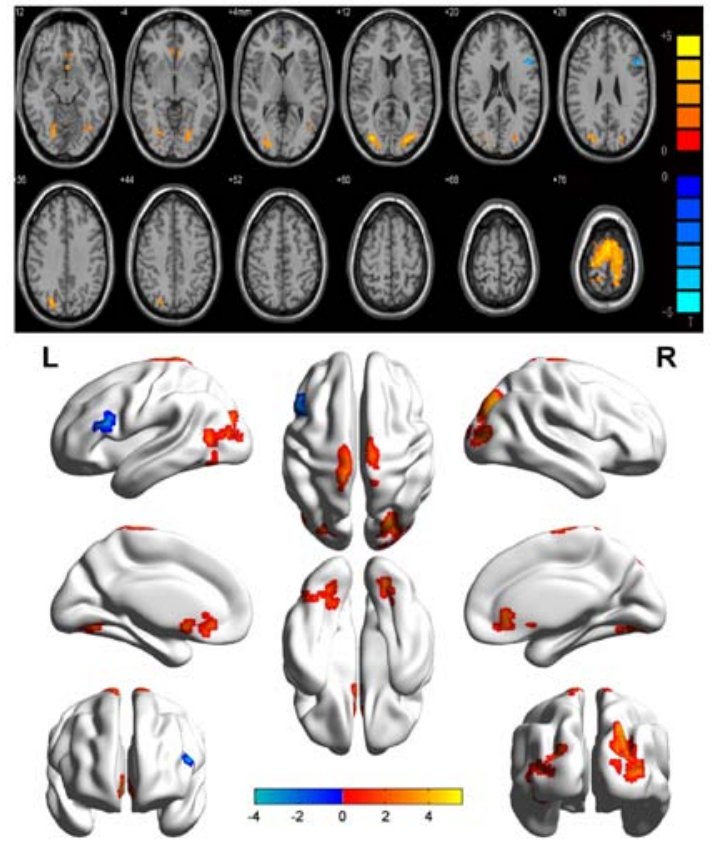

Figure 1. Spontaneous brain activity in patients with strabismus with amblyopia. Red regions (left lingual gyrus, right middle occipital gyrus/precuneus, bilateral anterior cingulate, left middle occipital gyrus and bilateral precentral gyrus) indicate higher ReHo values, while blue regions (left inferior frontal gyrus) represent lower ReHo values $(\mathrm{P}<0.05$; AlphaSim-corrected; cluster size, >40). ReHo, regional homogeneity; R, right; L, left.

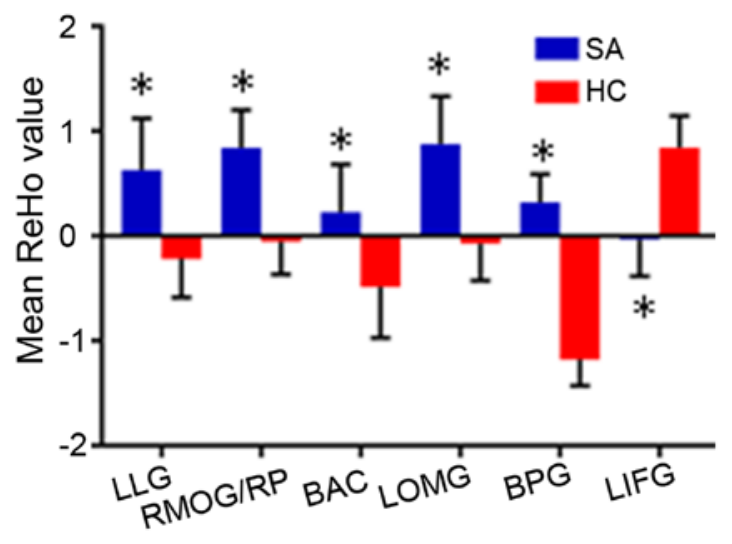

Figure 2. Mean ReHo values between the SA and HC groups in different areas of the brain. "P<0.05 vs. HC. ReHo, regional homogeneity; SA, strabismus and amblyopia; HC, healthy control; LLG, left lingual gyrus; RMOG/RP, right middle occipital gyrus/precuneus; BAC, bilateral anterior cingulate; LMOG, left middle occipital gyrus; BPG, bilateral precentral gyrus; LIFG, left inferior frontal gyrus.

Compared with the HC individuals, patients with SA exhibited significantly increased ReHo values in the LLG, RMOG/RP, BAC, LMOG and BPG areas, while the ReHo value for the LIFG region was significantly lower compared with that in HCs (Fig. 4). 

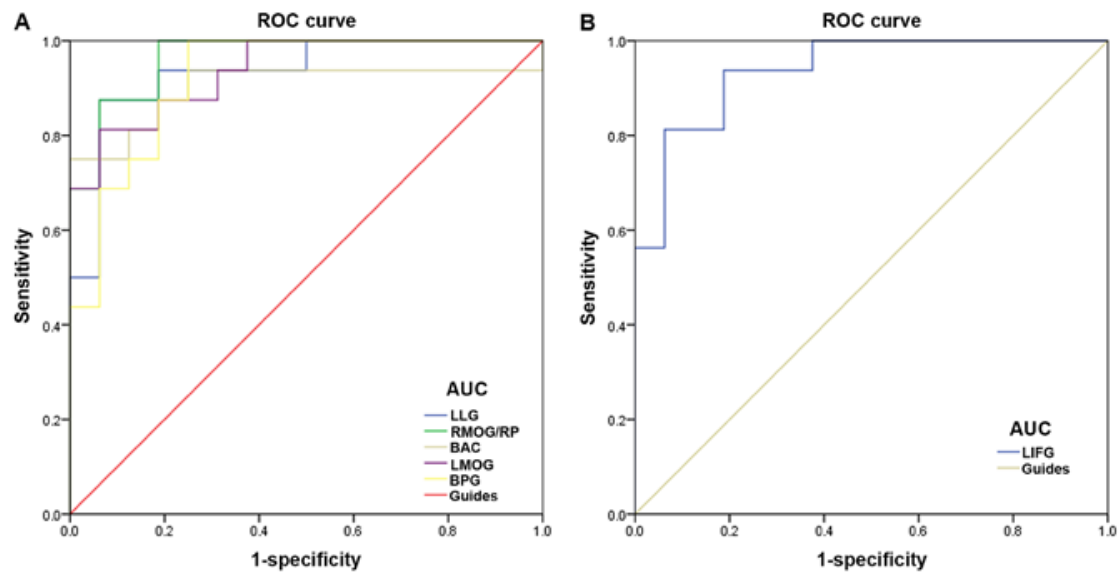

Figure 3. ROC curve analysis of the ReHo values for altered brain regions in the SA group. (A) The area under the ROC curve was 0.934 for LLG (P<0.001; 95\% CI, 0.847-1.000), 0.965 for RMOG/RP ( $\mathrm{P}<0.001 ; 95 \% \mathrm{CI}, 0.911-1.000), 0.902$ for $\mathrm{BAC}(\mathrm{P}<0.001 ; 95 \% \mathrm{CI}, 0.777-1.000), 0.938$ for LMOG $(\mathrm{P}<0.001$; 95\% CI, 0.860-1.000) and 0.922 for BPG (P<0.001; 95\% CI, 0.830-1.000). (B) Area under the ROC curve for LIFG was 0.938 (P<0.001; 95\% CI: 0.859-1.000). ROC, receiver operating characteristic; ReHo, regional homogeneity; SA, strabismus and amblyopia; LLG, left lingual gyrus; RMOG, right middle occipital gyrus; RP, right precuneus; BAC, bilateral anterior cingulate; LMOG, left middle occipital gyrus; BPG, bilateral precentral gyrus; LIFG, left inferior temporal gyrus.

The occipital lobe is a key brain area for visual processing, which controls eye movements and pupil accommodation reflex activities associated with vision. The lingual gyrus is part of the occipital lobe and an important component of the ventral visual stream, which participates in processing information such as shape, size, color, contour and object recognition. Thus, it is a crucial brain area for visual judgment and visual attention (32). Liang et al (33) used the voxel-mirrored homotopic connectivity (VMHC) method to analyze interhemispheric functional connectivity changes in patients with anisometropic amblyopia. They observed alterations in the lingual gyrus regions of patients with strabismus, amblyopia and anisometropic amblyopia, and the VMHC value of the lingual gyrus was found to be associated with stereoacuity. Furthermore, Qi et al (34) used the surface-based morphological measurement method and DTI to analyze changes in cortical thickness and white matter integrity in children with amblyopia. These researchers observed that the thickness of the lingual gyrus, cuneus and occipital cortex, and the fractional anisotropy (FA) values in the medial lingual cortex were all reduced. Yang et al (35) demonstrated that infants with esotropia exhibited high levels of activation in the lingual gyrus. Huang et al (19) also reported that the ReHo value of the lingual gyrus was increased in patients with concomitant strabismus. Similarly, the present study identified differences in the ReHo value of the LLG in adult patients with SA in comparison with the controls. This can be explained by visual compensation in patients with SA, while functional changes in the lingual gyrus may also cause visual impairment.

The cuneus is also located at the occipital lobe, which forms part of the visual center, and is involved in the processing of visual information in the retina-optic nerve-lateral geniculate pathway. Research by Schraa-Tam et al (36) demonstrated that the cuneus is involved in eye movement reflex that functions to stabilize the image of the retina; therefore, dysfunction of the cuneus causes eye movement disorders. The precuneus also forms an important part of the default mode network (DMN) of the brain, which is associated with cognition, memory, emotion

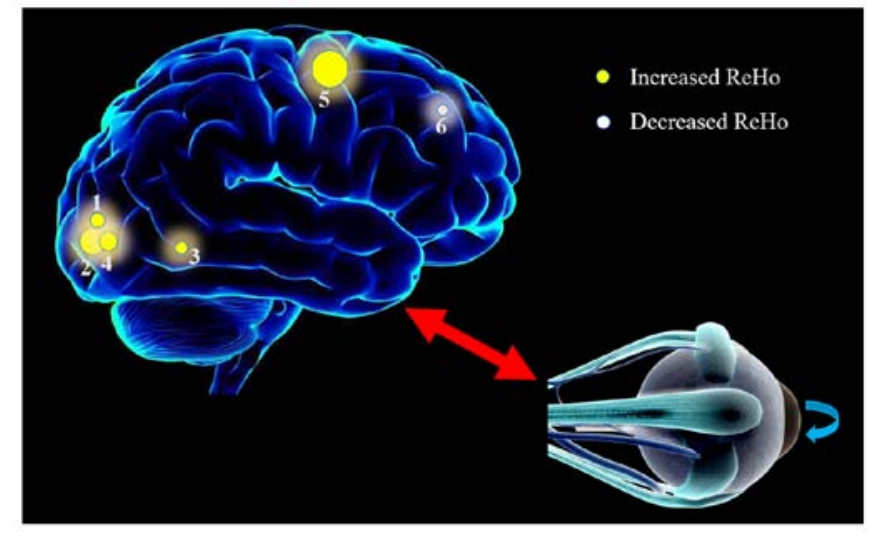

Figure 4. ReHo results of brain activity in the SA group. Compared with the healthy controls, the ReHo values of regions 1-5 in SA patients were increased to various extents, while the value of region 6 was decreased. Region 1 refers the left lingual gyrus (BA 19; $\mathrm{t}=3.6987$ ), region 2 to the right middle occipital gyrus/right precuneus (BA19; $t=4.7141$ ), region 3 to the bilateral anterior cingulate $(\mathrm{t}=3.8637)$, region 4 to the left middle occipital gyrus (BA 19; $\mathrm{t}=4.5112$ ), region 5 to the bilateral precentral gyrus (BA 6; $\mathrm{t}=5.5492$ ) and region 6 to left inferior frontal gyrus (BA9; $\mathrm{t}=-4.0693$ ). The size of the spots denotes the degree of quantitative change. ReHo, regional homogeneity; SA, strabismus and amblyopia; BA, Brodmann area.

and interaction regulation $(37,38)$. In a study of anisometropic amblyopia, Liang et al (39) reported decreased amplitude of low-frequency fluctuation values in the bilateral precuneus cortex in adults with anisometropic amblyopia, and the degree of reduction was associated with disease severity, suggesting that patients with bilateral precuneus neurons exhibited weaker activity. In addition, Huang et al (40) used DTI technology to analyze changes in whole brain microstructure in patients with strabismus. They found that the FA value of bilateral precuneus was significantly increased, suggesting enhanced fiber bundle integrity in this brain area. In the present study, the ReHo value of the right precuneus of adult SA patients was observed to be increased, which was similar to the results of Huang et al (40), but contrary to the findings presented by Liang et al (39). The differences observed among these studies may be attributed to 
variations among samples, leading to compensatory alterations to the precuneus. Simultaneously, lesions of the precuneus may also contribute to the SA occurrence.

The middle occipital gyrus and the lingual gyrus are in the $\mathrm{V} 2$ region, and the middle occipital gyrus is part of the dorsal visual stream (DVS) in the visual center (41). The DVS functions to analyze spatial information, such as location, direction, motion and action plan. Chan et al (17) used the voxel-based morphometry (VBM) method to analyze the volume of gray matter in patients with concomitant strabismus, and observed that the volume of gray matter in the strabismus group was decreased in the occipital and parietal lobes relative to the HCs. In addition, Yan et al (42) used DTI and VBM techniques to study the white matter structure in 13 patients with concomitant exotropia, and reported that the DVS was abnormal or impaired in these patients. By analyzing visual cortex changes, Jia et al (43) identified that patients with amblyopia exhibited significantly reduced changes in activation in the V2 visual area. In the current study, it was observed that ReHo values of the RMOG and LMOG in adult patients with SA were increased, which was different from the observations of previous studies. This may be due to differences in the samples included. The increased ReHo values observed in the present study can be attributed to visual compensation. According to evidence from earlier studies, changes in the middle occipital gyrus can be considered as one of the pathogenic factors in patients with SA.

The cingulate gyrus is located between the medial cingulate sulcus and the corpus callosum of the cerebral hemispheres (44), and is an important component of the limbic system and Papez circuits (45), as well as the DMN brain region, including the anterior cingulate gyrus, middle cingulate gyrus and posterior cingulate gyrus. The anterior cingulate gyrus has numerous established functions, including in emotion, cognition, movement, visceral movement, maternal behavior and social interactions. In addition, the association between the cingulate gyrus and epilepsy is currently a hot research topic $(46,47)$. A number of studies have noted that the anterior cingulate gyrus receives afferent neurons from the thalamus (48); therefore, it can be speculated that the cingulate gyrus may be associated with visual function. Zhai et al (49) used fMRI technology to analyze the efficacy of perceptual learning in the treatment of amblyopia, and reported that the primary visual cortex, visual junction cortex and right cingulate gyrus were significantly activated in patients receiving this treatment, suggesting that the cingulate gyrus contributes to the occurrence of amblyopia. In the present study, the results revealed that the ReHo value of the BAC gyrus in adult patients with SA was increased in comparison with that in the controls. The current study findings are similar to those reported by Zhai et al (49), suggesting that treatment of patients promotes compensation and that the cingulate gyrus is important in the occurrence of SA. Functional recovery of the cingulate gyrus can serve a compensatory role in treatment, which provides a further basis for the treatment of SA.

The frontal lobe is the most complex part of the brain. It mainly consists of four gyri, including the precentral, superior frontal, middle frontal and inferior frontal gyri. Frontal lobe lesions can cause several disorders, such as voluntary movement, speech, cranial nerve, autonomic nervous function and mental activity disorders. The precentral gyrus is also referred to as the 'cortical motor area', which mainly accepts proprioception from the skin, joints, tendons and skeletal muscles, and controls the voluntary movement of the body. However, the frontal lobe is also important to the integrity of visual function. Xiao et al (50) used VBM analysis to investigate changes in the visual cortex of amblyopic children, and identified that the gray matter density of the middle frontal gyrus was decreased. In a study of patients with strabismus, Ouyang et al (51) observed that the volumes of gray matter in the right posterior cingulate gyrus, precentral gyrus and left cuneus were decreased, while the white matter volume of the right precuneus and right anterior motor areas of the patients were significantly decreased. Furthermore, Huang et al (40) used DTI technology to analyze alterations in the whole brain microstructure in patients with strabismus, and reported that the mean diffusion coefficient in the left middle frontal gyrus was significantly decreased. In the current study, the data revealed that the ReHo values were lower in the inferior frontal gyrus of SA patients as compared with those in the HC group, which is consistent with the observations of previous studies, suggesting that the inferior frontal gyrus is important in the occurrence of SA. However, the current study results indicated that the ReHo value of the BPG was higher in SA patients in comparison with that in $\mathrm{HCs}$, which is contrary to the findings of previous studies. This is most likely a result of differences in the study populations. The current study results can be explained by a compensatory mechanism, while other studies have concluded that their findings can be explained by etiology. Nevertheless, all findings reveal that the frontal lobe serves a vital role in visual processing and associated eye movements.

ROC curve analysis in the present study demonstrated the accuracy of the ReHo method for the diagnosis of patients. Accuracy is perceived as excellent when AUC values are $0.7-0.9$, values between 0.5 and 0.7 are considered to indicate moderate accuracy, while values $<0.5$ indicate low discrimination between SA patients and HCs. The ROC curve values generated revealed that the AUC for each brain region was $>0.7$, indicating that the ReHo values of these changed brain regions exhibited a diagnostic accuracy for the identification of SA. Taken together, it is predicted that the ReHo method may be used for sensitive detection of SA in the future.

The present study also has certain limitations. The sample size was relatively small, and differences among samples may have impacted the results. Furthermore, for certain individuals, the length of the scanning time and small body movements may have affected the scan results.

In conclusion, the data of the present study demonstrated that patients with SA exhibit abnormal spontaneous activities in specific regions of the brain. These abnormal spontaneous activities may be associated with the occurrence of SA and are potentially associated with visual compensation. These findings provide a basis for the study of the pathogenesis of SA and indicate a potential direction for the development of treatment.

\section{Acknowledgements}

Not applicable. 


\section{Funding}

This study was supported by grants from the National Natural Science Foundation of China (grant nos. 81660158, 81460092 and 81400372); Jiangxi Natural Science Foundation major projects (grant no. 2016ACB21017); Jiangxi Province Key Research and Development Projects (grant no. 20151BBG70223, 20181BBG70004); Jiangxi Province Youth Science Foundation (grant nos. 20151BAB215016 and 20161BAB215198); Jiangxi Province Education Department Key Projects (grant no. GJ160020); Jiangxi Province Degree and Postgraduate Teaching Reform and Research Research Project (grant no. JXYJC2018-013); Spark Promotion Project for Appropriate Health Technologies at Grass-roots Level in Jiangxi Province (grant no. 20088003); Science and Technology Planning Project of Jiangxi Provincial Health Planning Commission (grant no. 20175116); Traditional Chinese Medicine Science and Technology Project of Jiangxi Provincial Health Planning Commission (grant no. 20150823).

\section{Availability of data and materials}

The datasets used and/or analyzed during the current study are available from the corresponding author on reasonable request.

\section{Authors' contributions}

YS, QHL and LY conceived and designed the study; QHL, BL, QL, TS, WQS, PWZ, QY, YQS, YH and WFL performed the experiments, and collected, analyzed and interpreted the data; QHL, BL, QL and TS wrote the study; YS. LY and QHL reviewed and edited the manuscript; all authors read and approved the manuscript.

\section{Ethics approval and consent to participate}

All procedures performed in the present study involving human participants were in accordance with the ethical standards of the Ethical Committee of the First Affiliated Hospital of Nanchang University (Nanchang, China), as well as with the 1964 Helsinki Declaration and its later amendments or comparable ethical standards. Informed consent was obtained from all individual participants included in the study.

\section{Patient consent for publication}

Not applicable.

\section{Competing interests}

The authors declare that they have no competing interests.

\section{References}

1. Chen X, Fu Z, Yu J, Ding H, Bai J, Chen J, Gong Y, Zhu H, Yu R and Liu H: Prevalence of amblyopia and strabismus in Eastern China: results from screening of preschool children aged 36-72 months. $\mathrm{Br}$ J Ophthalmol 100: 515-519, 2016.

2. Lewandowski KB: Strabismus as a possible sign of subclinical muscular dystrophy predisposing to rhabdomyolysis and myoglobinuria: a study of an affected family. Can Anaesth Soc J 29: 372-376, 1982.
3. Dickmann A, Petroni S, Salerni A, Parrilla R, Savino G, Battendieri R, Perrotta V, Radini C and Balestrazzi E: Effect of vertical transposition of the medial rectus muscle on primary position alignment in infantile esotropia with A- or V-pattern strabismus. J AAPOS 15: 14-16, 2011.

4. Clark RA: The role of extraocular muscle pulleys in incomitant nonparalytic strabismus. Middle East Afr J Ophthalmol 22: 279-285, 2015.

5. Oh SY, Clark RA, Velez F, Rosenbaum AL and Demer JL: Incomitant strabismus associated with instability of rectus pulleys. Invest Ophthalmol Vis Sci 43: 2169-2178, 2002.

6. Kekunnaya R, Negalur M. Duane retraction syndrome: causes, effects and management strategies. Clin Ophthal (Auckland, NZ); 11: 1917, 2017.

7. Breinin GM: In discussion of: De Gindersen T, Zeavin B Observations on the retraction syndrome of Duane. Arch Ophthalmol 55: 576, 1956.

8. Parsa CF, Grant PE, Dillon WP Jr, du Lac S and Hoyt WF: Absence of the abducens nerve in Duane syndrome verified by magnetic resonance imaging. Am J Ophthalmol 125: 399-401, 1998.

9. Lueder GT, Dunbar JA, Soltau JB, Lee BC and McDermott M: Vertical strabismus resulting from an anomalous extraocular muscle. J AAPOS 2: 126-128, 1998.

10. Zhao K and Shi X: Learn new version of Preferred Practice Pattern to further standardize the diagnosis and treatment of amblyopia. Zhonghua Yan Ke Za Zhi 50: 481-484, 2014. (In Chinese).

11. Jin H, Yi JL, Xie H, Xiao F, Wang WJ, Shu XM, Xu YL, Chen SL and Ye WX: A study on visual development among preschool children. Zhonghua yan ke za Zhi 47: 1102-1106, 2011.

12. Avram E and Stănilă A: Functional amblyopia. Oftalmologia 57: 3-8, 2013 (In Romanian).

13. von Noorden GK: Abnormal Binocular Interaction: Evidence in Humans. In: Strabismus and Amblyopia. Lennerstrand G, von Noorden GK, Campos EC (eds). Wenner-Gren Center International Symposium Series, Palgrave Macmillan, London, pp 275-284, 1988.

14. Sjöstrand JB: Form deprivation amblyopia-a treatable cause of blindness. Acta Ophthalmol 86: s243, 2010.

15. Wang H, Crewther SG, Liang M, Laycock R, Yu T, Alexander B, Crewther DP, Wang J, Yin Z: Impaired activation of visual attention network for motion salience is accompanied by reduced functional connectivity between frontal eye fields and visual cortex in Strabismic Amblyopia. Front Hum Neurosci 11: 195, 2017.

16. Turner R: Functional Magnetic Resonance Imaging (fMRI). In: Runehov A.L.C., Oviedo L. (eds) Encyclopedia of Sciences and Religions 2013: 35, 2013.

17. Chan ST, Tang KW, Lam KC, Chan LK, Mendola JD and Kwong KK: Neuroanatomy of adult strabismus: A voxel-based morphometric analysis of magnetic resonance structural scans. Neuroimage 22: 986-994, 2004.

18. Bi H, Zhang B, Tao X, Harwerth RS, Smith EL III and Chino YM: Neuronal responses in visual area V2 (V2) of macaque monkeys with strabismic amblyopia. Cereb Cortex 21: 2033-2045, 2011.

19. Huang X, Li SH, Zhou FQ, Zhang Y, Zhong YL, Cai FQ, Shao Y and Zeng XJ: Altered intrinsic regional brain spontaneous activity in patients with comitant strabismus: a resting-state functional MRI study. Neuropsychiatr Dis Treat 12: 1303-1308, 2016.

20. Zang Y, Jiang T, Lu Y, He Y and Tian L: Regional homogeneity approach to fMRI data analysis. Neuroimage 22: 394-400, 2004.

21. Tononi G, McIntosh AR, Russell DP and Edelman GM: Functional clustering: Identifying strongly interactive brain regions in neuroimaging data. Neuroimage 7: 133-149, 1998.

22. Shao Y, Cai FQ, Zhong YL, Huang X, Zhang Y, Hu PH, Pei CG, Zhou FQ and Zeng XJ: Altered intrinsic regional spontaneous brain activity in patients with optic neuritis: a resting-state functional magnetic resonance imaging study. Neuropsychiatr Dis Treat 11: 3065-3073, 2015.

23. Cui Y, Jiao Y, Chen YC, Wang K, Gao B, Wen S, Ju S and Teng GJ: Altered spontaneous brain activity in type 2 diabetes: a resting-state functional MRI study. Diabetes 63: 749-760, 2014.

24. Song Y, Mu K, Wang J, Lin F, Chen Z, Yan X, Hao Y, Zhu W and Zhang H: Altered spontaneous brain activity in primary open angle glaucoma: a resting-state functional magnetic resonance imaging study. PLoS One 9: e89493, 2014.

25. Huang X, Li HJ, Ye L, Zhang Y, Wei R, Zhong YL, Peng DC and Shao Y: Altered regional homogeneity in patients with unilateral acute open-globe injury: a resting-state functional MRI study. Neuropsychiatr Dis Treat 12: 1901-1906, 2016. 
26. Huang X, Ye CL, Zhong YL, Ye L, Yang QC, Li HJ, Jiang N, Peng DC and Shao Y: Altered regional homogeneity in patients with late monocular blindness: a resting-state functional MRI study. Neuroreport 28: 1085-1091, 2017.

27. Huang X, Li D, Li HJ, Zhong YL, Freeberg S, Bao J, Zeng XJ and Shao Y: Abnormal regional spontaneous neural activity in visual pathway in retinal detachment patients: a resting-state functional MRI study. Neuropsychiatr Dis Treat 13: 2849-2854, 2017.

28. Dai XJ, Gong HH, Wang YX, Zhou FQ, Min YJ, Zhao F, Wang SY, Liu BX and Xiao XZ: Gender differences in brain regional homogeneity of healthy subjects after normal sleep and after sleep deprivation: a resting-state fMRI study. Sleep Med 13: 720-727, 2012

29. Li Y, Liang P, Jia $X$ and Li K: Abnormal regional homogeneity in Parkinson's disease: a resting state fMRI study. Clin Radiol 71: e28-e34, 2016.

30. Holmes D, Rettmann M and Robb R: Visualization in ImageGuided Interventions. In: Image-Guided Interventions. Peters T and Cleary K (eds). Springer, Boston, MA. 2008.

31. Harrison BJ and Pantelis C: Gradient-Echo Images. In: Encyclopedia of Psychopharmacology. Stolerman IP (ed) Springer, Berlin, Heidelberg, 2010.

32. Lee HW, Hong SB, Seo DW, Tae WS and Hong SC: Mapping of functional organization in human visual cortex: electrical cortical stimulation. Neurology 54: 849-854, 2000.

33. Liang M, Xie B, Yang H, Yin X, Wang H, Yu L, He S and Wang J: Altered interhemispheric functional connectivity in patients with anisometropic and strabismic amblyopia: a resting-state fMR study. Neuroradiology 59: 517-524, 2017.

34. Qi S, Mu YF, Cui LB, Li R, Shi M, Liu Y, Xu JQ, Zhang J, Yang J and Yin $\mathrm{H}$ : Association of optic radiation integrity with cortical thickness in children with Anisometropic Amblyopia. Neurosci Bull 32: 51-60, 2016.

35. Yang X, Zhang J, Lang L, Gong Q and Liu L: Assessment of cortical dysfunction in infantile esotropia using fMRI. Eur J Ophthalmol 24: 409-416, 2014.

36. Schraa-Tam CK, van der Lugt A, Smits M, Frens MA, van Broekhoven PC and van der Geest JN: Differences between smooth pursuit and optokinetic eye movements using limited lifetime dot stimulation: a functional magnetic resonance imaging study. Clin Physiol Funct Imaging 29: 245-254, 2009.

37. Uddin LQ: Salience processing and insular cortical function and dysfunction. Nat Rev Neurosci 16: 55-61, 2015.

38. Weissman-Fogel I, Moayedi M, Taylor KS, Pope G and Davis KD Cognitive and default-mode resting state networks: Do male and female brains "rest" differently? Hum Brain Mapp 31: 1713-1726, 2010.

39. Liang M, Xie B, Yang H, Yu L, Yin X, Wei L and Wang J: Distinct patterns of spontaneous brain activity between children and adults with anisometropic amblyopia: a resting-state fMRI study. Graefes Arch Clin Exp Ophthalmol 254: 569-576, 2016.
40. Huang X, Li H-J, Zhang Y, Peng DC, Hu PH, Zhong YL, Zhou FQ and Shao Y: Microstructural changes of the whole brain in patients with comitant strabismus: evidence from a diffusion tensor imaging study. Neuropsychiatr Dis Treat 12: 2007-2014, 2016.

41. Wandell BA, Dumoulin SO and Brewer AA: Visual field maps in human cortex. Neuron 56: 366-383, 2007.

42. Yan X, Lin X, Wang Q, Zhang Y, Chen Y, Song S, Jiang T: Doral visual pathway changes in patients with comitant extropia. PLoS ONE 5 (6): e10931, 2010.

43. Jia CH, Lu GM, Zhang ZQ, Wang Z, Huang W, Ma F, Yin J, Huang ZP and Shao Q: Comparison of deficits in visual cortex between anisometropic and strabismic amblyopia by fMRI retinotopic mapping. Zhonghua Yi Xue Za Zhi 90: 1446-1452, 2010 (In Chinese).

44. San Pedro EC, Mountz JM, Ojha B, Khan AA, Liu HG and Kuzniecky RI: Anterior cingulate gyrus epilepsy: the role of ictal rCBF SPECT in seizure localization. Epilepsia 41: 594-600, 2000.

45. Devinsky O, Morrell M, Vogt BA: Contribution of anterior cingulate cortex to behavior. Brain 118 (Pt 1): 279-306, 1995.

46. Braga AMDS, Fujisao EK, Verdade RC, Paschoalato RP, Paschoalato RP, Yamashita S and Betting LE: Investigation of the cingulate cortex in idiopathic generalized epilepsy. Epilepsia 56: 1803-1811, 2015 .

47. Alkawadri R, So NK, Van Ness PC and Alexopoulos AV: Cingulate epilepsy: report of 3 electroclinical subtypes with surgical outcomes. JAMA Neurol 70: 995-1002, 2013.

48. Unnwongse K, Wehner T and Foldvary-Schaefer N: Mesial frontal lobe epilepsy. J Clin Neurophysiol 29: 371-378, 2012.

49. Zhai J, Chen M, Liu L, Zhao X, Zhang H, Luo X and Gao J: Perceptual learning treatment in patients with anisometropic amblyopia: a neuroimaging study. Br J Ophthalmol 97: 1420-1424, 2013

50. Xiao JX, Xie S, Ye JT, Liu HH, Gan XL, Gong GL and Jiang XX: Detection of abnormal visual cortex in children with amblyopia by voxel-based morphometry. Am J Ophthalmol 143: 489-493, 2007.

51. Ouyang J, Yang L, Huang X, Zhong YL, Hu PH, Zhang Y, Pei CG and Shao Y: The atrophy of white and gray matter volume in patients with comitant strabismus: evidence from a voxel-based morphometry study. Mol Med Rep 16: 3276-3282, 2017.

This work is licensed under a Creative Commons Attribution-NonCommercial-NoDerivatives 4.0 International (CC BY-NC-ND 4.0) License. 